\title{
Limites de la modélisation bidimensionnelle des tunnels urbains pour la prévision des tassements
}

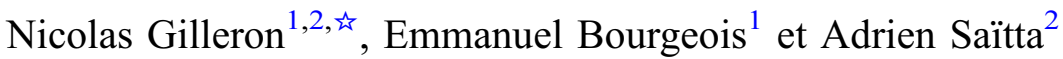 \\ 1 Université Paris-Est/IFSTTAR/COSYS, Champs-Sur-Marne, France \\ 2 EGIS, Saint-Quentin-en-Yvelines, France
}

\begin{abstract}
Résumé - La prévision des tassements provoqués en surface par le creusement de tunnels peu profonds repose largement sur le recours à des modèles numériques bidimensionnels, en s'appuyant sur la méthode convergence-confinement. Dans cet article, on cherche à mettre en évidence les limites de cette approche. On définit une situation caractéristique du creusement d'un tunnel en région parisienne, et on cale le taux de déconfinement d'un modèle bidimensionnel afin d'obtenir le même tassement maximal que pour le modèle tridimensionnel équivalent. Une fois ce calage effectué, on s'intéresse au profil des invariants de contraintes. L'évolution de la contrainte déviatorique au-dessus de la voûte du tunnel, avant la mise en place du soutènement, est opposée selon la modélisation. Ce résultat explique pourquoi le taux de déconfinement dépend fortement de la loi de comportement et ne peut être estimé a priori dans le cas de lois de comportement avancées. L'illustration avec trois lois de comportement différentes, une loi élasto-plastique parfaite, une loi élastique non linéaire et le Hardening Soil Model met en évidence les limites très significatives de la modélisation bidimensionnelle de tunnels pour la prévision des tassements.
\end{abstract}

Mots clés : tunnel / éléments finis / tassement / convergence-confinement

\begin{abstract}
Limits of 2D modelling of urban tunneling. The prediction of settlements caused by shallow tunnelling is mainly based on two-dimensional modelling with the stress relaxation method. In this article, we illustrate some limits of this method. We define a characteristic tunnel typical for the Paris region, then we adjust the relaxation factor of a 2D model to have the same maximum settlement as in the equivalent 3D model. Once this fitting is done, we look at the stress invariants paths. The evolution of the deviatoric stress above the tunnel crown, before the setting of the lining, is opposed according to the considered simulation. This result explains why the stress relaxation factor depends strongly on the constitutive model. Furthermore, it shows that the use of a 2D model for a stress analysis of the soil is uncertain. The discussion is illustrated by simulations carried out with three different constitutive models: a perfect elasto-plastic, a non-linear elastic and the Hardening Soil Model.
\end{abstract}

Keywords: tunnel / finite element / settlement / stress relaxation method

\section{Points importants}

On explicite la difficulté du calage du taux de déconfinement pour la prévision des tassements. On met en évidence des différences dans l'état de contraintes dans le massif entre les modèles 2D et 3D avant pose du soutènement par des figures originales. On illustre sur trois lois de comportements dont une, non linéaire, est originale et a été développée pour ce sujet.

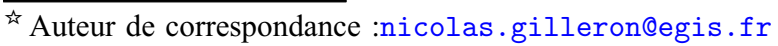

\section{Introduction}

La prévision des tassements induits par le creusement des tunnels urbains est un enjeu essentiel de ces projets. Malgré l'augmentation des capacités de calcul et le caractère fortement tridimensionnel du problème, les modélisations bidimensionnelles sont encore largement plébiscitées par la profession. Selon un sondage réalisé par l'AITES en 2012, $85 \%$ des analyses numériques sont bidimensionnelles et parmi celles-ci $65 \%$ sont réalisées à l'aide de modèles élastiques linéaires avec critère de rupture de Mohr-Coulomb (Wedekin et al., 2012). La méthode bidimensionnelle la plus courante consiste à déterminer le taux de déconfinement du terrain à la pose du 
soutènement à l'aide de la méthode convergence-confinement. Il n'existe pas de consensus sur la manière de déterminer ce taux dans le cadre de l'étude de tunnels urbains. Afin d'éclairer cette difficulté, on étudie ici les différences entre les états de contraintes dans le massif obtenus avec les modélisations bidimensionnelles et tridimensionnelles. On met alors en évidence les limites des modélisations bidimensionnelles. Nous illustrons ensuite ce propos avec une modélisation élasto-plastique parfaite, une loi élastique non linéaire originale et le Hardening Soil Model. Les modélisations numériques ont été faites à l'aide de CESAR-LCPCv6 (Humbert et al., 2005) et Plaxis 2015 pour celles utilisant le HSM.

\section{Modèles numériques de tunnels urbains}

\subsection{Caractéristiques du tunnel}

On considère un tunnel circulaire de $10 \mathrm{~m}$ de diamètre, excavé selon la méthode conventionnelle dans un massif homogène. La profondeur de l'axe est de $20 \mathrm{~m}$. Le soutènement est constitué de béton projeté et de cintres métalliques : il est mis en place à $2 \mathrm{~m}$ en arrière du front de taille. Ces valeurs sont élevées pour un tunnel excavé en méthode conventionnelle : elles nous permettent d'avoir des illustrations plus frappantes, mais les conclusions seraient identiques avec un plus petit diamètre et une plus petite distance non supportée.

Le soutènement fait $30 \mathrm{~cm}$ d'épaisseur et son module de Young est pris égal à $10 \mathrm{GPa}$ avec un coefficient de Poisson égal à 0,2 .

L'extension transversale des modèles numériques est égale à $50 \mathrm{~m}$, les déplacements sont bloqués à $35 \mathrm{~m}$ de profondeur, modélisant un substratum rocheux ou une couche de sol significativement plus raide.

Le poids volumique du massif est égal à $20 \mathrm{kN} / \mathrm{m}^{3}$. Le coefficient de pression des terres au repos est égal à 0,7 . Ce coefficient a une importance significative dans les résultats présentés ci-après. Une valeur comprise entre 0,5 et 0,9 est représentative des sols sédimentaires.

\subsection{Modélisation du cycle d'excavation}

En modélisation bidimensionnelle, on se place dans le cadre des déformations planes dans le plan transversal à l'axe du tunnel. La méthode convergence-confinement (Panet, 1995) est largement utilisée pour la modélisation 2D du creusement. On rend compte de la convergence progressive du terrain en avant du front et en arrière du front par une « relaxation » des contraintes appliquées par la zone excavée sur le terrain restant. La méthode repose sur le choix d'un taux de déconfinement qui a pour objet de tenir compte de la déformation du terrain qui s'est produite avant la mise en place du soutènement. La modélisation s'effectue en deux étapes : dans la première, la raideur du soutènement n'est pas prise en compte, et on applique sur le contour de la zone excavée des forces de déconfinement égales aux forces surfaciques exercées dans le massif non perturbé par la zone excavée sur le massif restant en place, multipliées par le taux de déconfinement $\lambda$; dans la seconde, après activation $\mathrm{du}$ soutènement, le reste de l'effort (égal aux forces surfaciques initiales multipliées par $(1-\lambda))$ est appliqué. Cette méthode présente l'avantage d'être relativement facile à mettre en œuvre

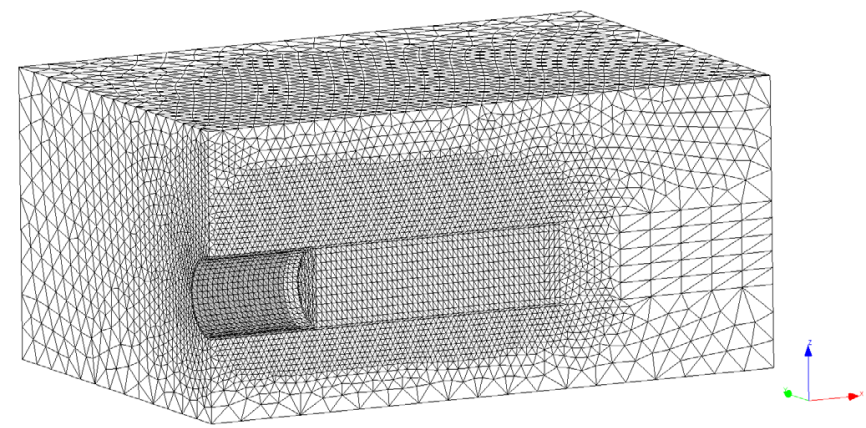

Fig. 1. Modèle 3D pour la simulation du creusement d'un tunnel par la méthode conventionnelle.

Fig. 1. 3D Model of conventional tunneling.

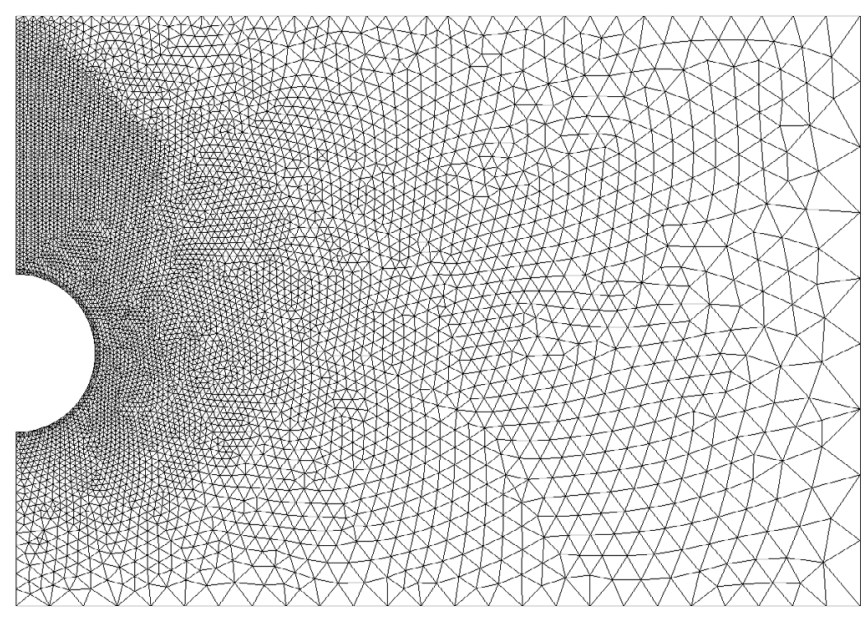

Fig. 2. Modèle 2D pour la simulation du creusement d'un tunnel par la méthode conventionnelle.

Fig. 2. 2D Model of conventional tunneling.

(y compris analytiquement) et de respecter l'équilibre statique des forces.

En modélisation tridimensionnelle, il faut définir l'extension longitudinale du modèle et le nombre de passes d'excavations modélisées. L'extension longitudinale est la somme de la longueur excavée, multiple du pas d'excavation, et de la longueur entre le front d'excavation et le bord du maillage : cette longueur doit être suffisante pour que le résultat ne soit pas influencé par ce bord. On retient ici 16 phases d'excavation et $42 \mathrm{~m}$ en avant de la position finale du front, ce qui donne une extension longitudinale totale du modèle égale à $74 \mathrm{~m}$. Le tassement maximal est calculé à partir de la contribution de la seule dernière phase d'excavation aux déplacements selon la méthode décrite par Möller et al. (2003). On s'affranchit ainsi des conditions de bords dans le sens longitudinal.

Les maillages utilisés sont présentés sur les Figures 1 et 2 .

\section{Des écarts conséquents selon la méthode de modélisation}

\subsection{Calage du taux de déconfinement}

Pour étudier l'évolution des contraintes, on considère dans cette partie un massif homogène avec un comportement élastique linéaire (module de Young $E=100 \mathrm{MPa}$, coefficient 
Tableau 1. Tassement maximal en fonction du taux de déconfinement.

Table 1. Maximum settlement versus stress relaxation factor.

\begin{tabular}{llllllll}
\hline$\lambda(\%)$ & 20 & 28 & 30 & 40 & 50 & 60 & 70 \\
$S_{i}(\mathrm{~mm})$ & 3,1 & 4,3 & 4,6 & 6,1 & 7,6 & 9,2 & 10,7 \\
$S_{\max }(\mathrm{mm})$ & 3,9 & 5,1 & 5,3 & 6,7 & 8,2 & 9,6 & 11 \\
\hline
\end{tabular}

de Poisson $v=0,2$ ). Nous avons effectué des études paramétriques qui montrent que les taux de déconfinement et profils de contraintes obtenus sont peu sensibles au module de Young, sur une plage 20-200 MPa et au coefficient de Poisson, sur une plage 0,15-0,35.

Le tassement maximal issu du calcul tridimensionnel avec le modèle élastique linéaire est de $5,1 \mathrm{~mm}$. En clé, le déplacement vertical maximal est de $10,5 \mathrm{~mm}$. Il atteint $30 \%$ de cette valeur au front de taille (préconvergence) et $80 \%$ de cette valeur à la pose du soutènement: dans le calcul, l'essentiel du tassement se produit avant la pose du soutènement et le soutènement joue bien son rôle de stabilisation de l'excavation et de blocage des déplacements.

Dans la modélisation bidimensionnelle, le tassement maximal est une fonction du taux de déconfinement. On présente dans le Tableau 1 le tassement maximal à l'issue de la première phase du calcul $S_{i}$ et le tassement à la fin du calcul après pose du soutènement $S_{\max }$ en fonction du taux de déconfinement $\lambda$. L'écart entre $S_{i}$ et $S_{\max }$ varie de $+25 \%$ (pour $\lambda=20 \%$ ) à $+2 \%$ (pour $\lambda=70 \%$ ). Comme dans la modélisation tridimensionnelle, le tassement se produit majoritairement avant la pose du soutènement.

$\mathrm{Si}$ on détermine le taux de déconfinement à la mise en œuvre du soutènement par calage du tassement maximal sur le modèle tridimensionnel, on obtient un taux de déconfinement égal à $28 \%$. La détermination de $\lambda$ par calage d'un modèle $2 \mathrm{D}$ sur les résultats d'un modèle 3D (le tassement ou d'autres grandeurs) est une pratique courante dans l'ingénierie (Janin et al., 2015 par exemple). On pourrait également proposer de caler le taux de déconfinement à partir du volume de la cuvette, mais ce calage est moins direct. D'autre part, dans les simulations présentées ici, la largeur de la cuvette est relativement peu sensible aux paramètres rhéologiques qu'on retient, de sorte que les variations du volume perdu en surface sont très similaires à celles du tassement maximal.

À titre de comparaison, on peut calculer le taux de déconfinement à la pose du soutènement au moyen des formules présentées dans la référence Panet (1995). Elles ont été établies sur la base de modèles numériques axisymétriques et sont donc plutôt adaptées au cas où le tunnel est profond et où l'état de contraintes initial est homogène et isotrope. Ces corrélations ont été établies par calage de la convergence radiale de la section. On peut considérer par exemple l'équation (1) :

$$
\lambda=\alpha_{0}+\left(1-\alpha_{0}\right)\left[1-\left(\frac{m R}{m R+d}\right)^{2}\right] \text {, }
$$

où $\alpha_{0}$ et $m$ sont deux paramètres sans dimension, $R$ est le diamètre du tunnel et $d$ la distance entre le point considéré et le front. Panet (1995) recommande de prendre $\alpha_{0}=0,25$ et $m=0,75$. Par ailleurs, dans notre situation, on a $d=2 \mathrm{~m}$ et $R=5 \mathrm{~m}$. Le taux de déconfinement calculé est alors de $68 \%$.
Pour cette valeur, le tassement maximal du modèle bidimensionnel est plus de deux fois supérieur au tridimensionnel.

Évidemment, les hypothèses adoptées ici sont différentes de celles de Panet (1995) où la géométrie est différente et où le calage est fait sur la convergence radiale. L'écart entre les taux de déconfinement obtenus met bien en évidence ces différences, et illustre le risque que présente l'utilisation d'une formule hors du domaine pour lequel elle a été établie. Des corrections à l'équation (1) ont été proposées pour tenir compte de la raideur du soutènement par exemple Bernaud et Rousset (1992) mais, malgré ces corrections, le taux de déconfinement reste environ deux fois supérieur à celui obtenu par calage du tassement maximum sur le résultat du calcul tridimensionnel.

Dans la suite, on propose une étude comparée des contraintes, et on se concentre sur les étapes en amont de la pose du soutènement, là où se produit la plus grande partie des tassements.

\section{2 Évolution des contraintes dans la modélisation bidimensionnelle}

On définit classiquement la contrainte moyenne effective $p$ ' et la contrainte déviatorique $q$ par :

$$
\begin{gathered}
p^{\prime}=\frac{1}{3}\left(\sigma_{1}^{\prime}+\sigma_{2}^{\prime}+\sigma_{3}^{\prime}\right), \\
q=\sqrt{\frac{1}{2}\left[\left(\sigma_{1}-\sigma_{2}\right)^{2}+\left(\sigma_{1}-\sigma_{3}\right)^{2}+\left(\sigma_{3}-\sigma_{1}\right)^{2}\right]} .
\end{gathered}
$$

On représente sur la Figure 3 les isovaleurs de $p$ ' et de $q$ calculées en 2D et normalisées par leur valeur initiale respective, en fonction du taux de déconfinement $(10 ; 20$ et $28 \%$ ) et à la fin de la dernière étape du calcul (déconfinement avec soutènement en place).

Les zones où l'invariant est compris entre 0,9 et 1,1 sont laissées blanches. Un dégradé de rouge indique une valeur supérieure, les transitions entre couleurs correspondent aux valeurs 1,$5 ; 2$ et 3 . Un dégradé de bleu indique une valeur inférieure, les transitions entre couleurs correspondent aux valeurs 0,8 et 0,6 .

La contrainte moyenne dans le massif reste proche de sa valeur initiale sur toutes les phases d'excavation. On retrouve ici le résultat classique du calcul élastique linéaire pour un état de contraintes initiales homogènes et isotropes (dans un massif infini), où la contrainte moyenne est strictement constante. Pour la contrainte déviatorique, on observe une évolution jusqu'à la mise en place du soutènement : dans la zone audessus du tunnel, la contrainte déviatorique diminue puis se stabilise à environ $50 \%$ de sa valeur initiale ; en rein, elle augmente dès la première phase de creusement jusqu'à doubler à la paroi. Ce résultat est en revanche nettement différent du cas où les contraintes initiales sont homogènes et isotropes, pour lequel la contrainte déviatorique augmente à la paroi, et il n'y a pas de différence entre la clé et le rein. Ainsi, l'anisotropie des contraintes initiales explique la différence entre l'évolution en rein et en voûte. En voûte, la contrainte radiale est la contrainte verticale, qui est supérieure à la contrainte horizontale à l'état initial : comme la contrainte verticale diminue, la contrainte déviatorique diminue. 


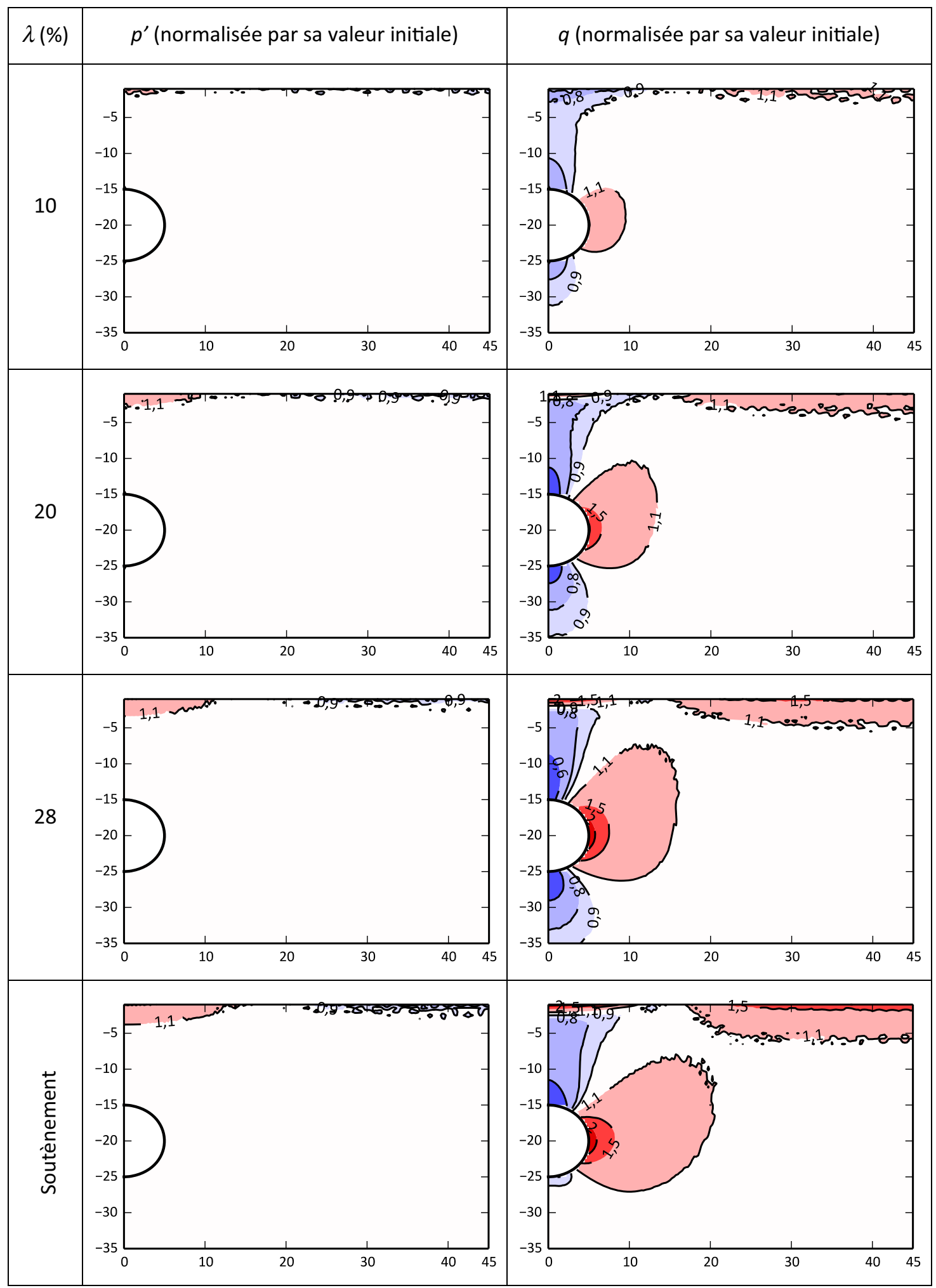

Fig. 3. Évolution des invariants de contraintes en fonction du taux de déconfinement.

Fig. 3. Evolution of stress invariants with stress relaxation factor.

Après la mise en place du soutènement, le calcul donne des variations de contraintes marginales en rein et au-dessus de la voûte. En radier, on note un retour au niveau initial de la contrainte déviatorique dû à la proximité de la condition limite inférieure.

\section{3 Évolution des contraintes dans la modélisation tridimensionnelle}

Dans la représentation classique de la méthode convergence-confinement, on associe à une valeur du taux de 


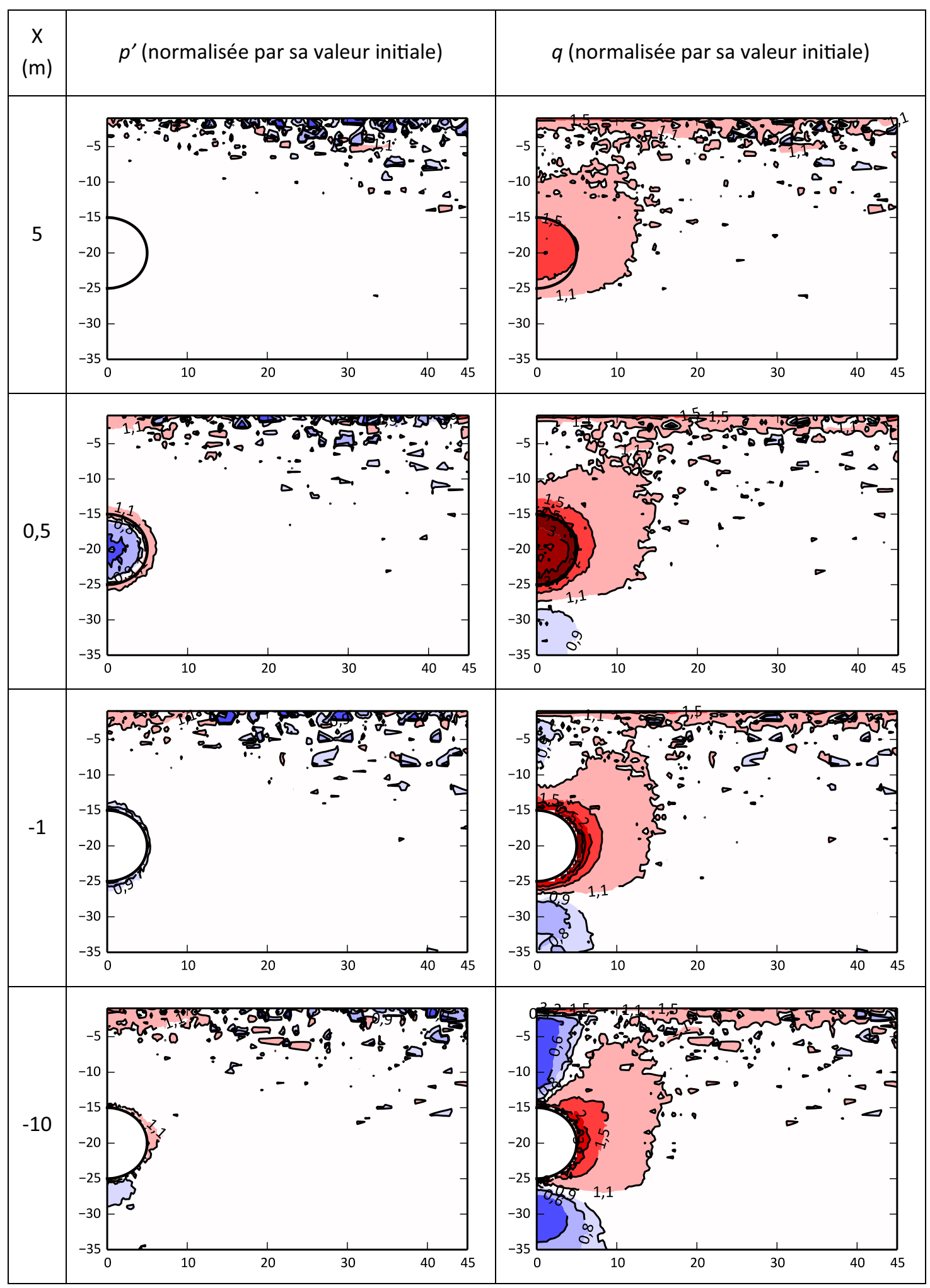

Fig. 4. Évolution des invariants de contraintes en fonction de la distance au front.

Fig. 4. Evolution of stress invariants in $3 D$ with distance from excavation face.

déconfinement une distance au front de taille (par exemple dans Éq. (1) $\alpha_{0}$ est le taux de déconfinement pour $d=0$, donc au front de taille). On présente sur la Figure 4 les isovaleurs des invariants de contraintes dans des coupes transversales au modèle 3D, pour différentes distances au front de taille. On cherche ainsi à évaluer si on peut associer, comme dans la représentation classique, une distance au front de taille à un taux de déconfinement mais sur la base des invariants de 


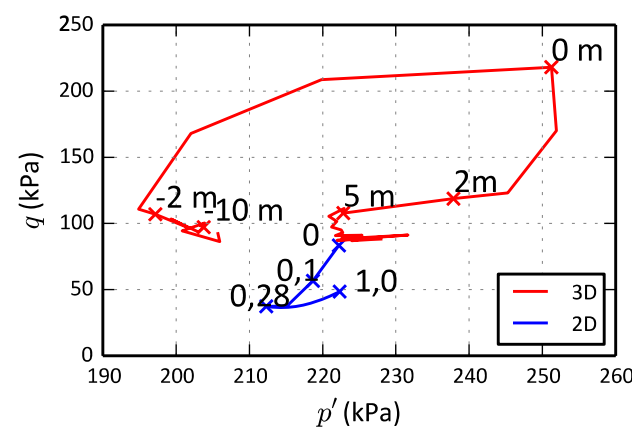

(a)

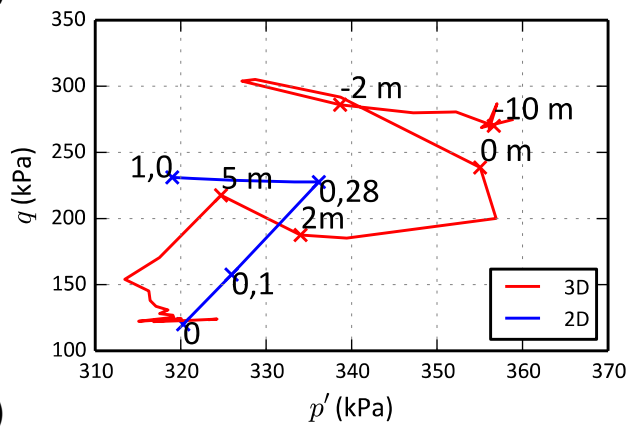

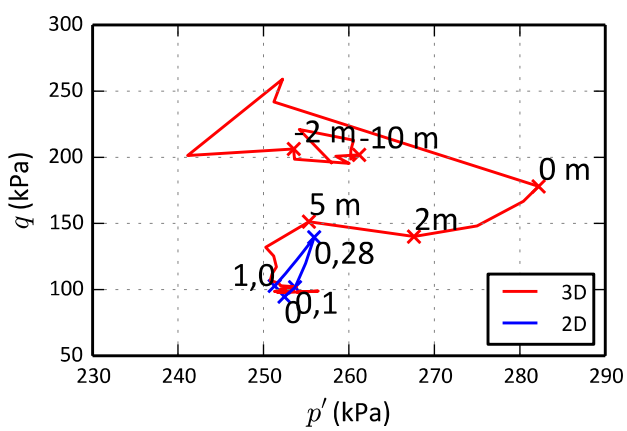

(b)

Fig. 5. Chemin de contraintes dans le plan $\left(p^{\prime}, q\right)$ en fonction du taux de déconfinement en $2 \mathrm{D}$ et de la distance au front de taille en 3D à $1 \mathrm{~m}$ de la section (a) en clé (b) à $45^{\circ}$ de l'axe vertical (c) en rein.

Fig. 5. Stress path in $\left(p^{\prime}, q\right)$ plan versus stress relaxation factor in $2 D$ and distance to the excavation face in $3 D$ at $1 \mathrm{~m}$ from section (a) in crown (b) $45^{\circ}$ from vertical axis (c) in sidewall.

contraintes et non de la convergence en clé. Les conventions de couleurs sont les mêmes que sur la Figure 3.

On distingue l'avant du front $(X>0)$, la zone du front de taille (valeurs de $\mathrm{X}$ voisines de 0 ) et la zone supportée (X $<-2 \mathrm{~m}$ ). Comme dans le calcul 2D, la contrainte moyenne dans l'ensemble du massif reste généralement proche de sa valeur initiale, sauf près du front dans la zone du noyau d'excavation. En avant du front et jusqu'à la mise en place du soutènement, la contrainte déviatorique est très largement supérieure à sa valeur initiale en rein mais aussi au-dessus de la clé (entre 1,5 et 2 fois la valeur initiale). Dans la zone soutenue derrière le front $(X=-10 \mathrm{~m})$, la contrainte déviatorique en rein est proche de sa valeur à la pose du soutènement, le déviateur des contraintes en clé et en radier diminue jusqu'à être $40 \%$ inférieur à sa valeur initiale.

\subsection{Différences et conséquences}

Pour les deux modélisations, la contrainte moyenne est quasi constante dans le massif. Concernant la contrainte déviatorique, la situation est plus compliquée. En avant du front de taille et jusqu'à la pose du soutènement $(X>-2 \mathrm{~m}$ et $\lambda=0,28)$, le profil est très différent entre les modélisations : alors que dans le modèle bidimensionnel la contrainte déviatorique est largement inférieure à sa valeur initiale en radier et en voûte, elle est supérieure à sa valeur initiale audessus de la voûte dans la modélisation tridimensionnelle. Finalement, les profils des invariants de contraintes 3D loin du front et $2 \mathrm{D}$ à la fin de la dernière étape de calcul avec soutènement sont qualitativement assez proches dans le massif mais les valeurs obtenues sont sensiblement différentes à la paroi. On illustre la différence dans les chemins de contraintes aux abords de la section sur la Figure 5. Comme la comparaison des Figures 3 et 4, elle montre qu'on ne peut pas corréler la distance au front de taille et le taux de déconfinement sur les invariants de contraintes $p$ ' et $q$. Les considérations qui précèdent reposent sur des calculs élastiques linéaires. On fait l'hypothèse, vérifiée a posteriori, que les chemins de contraintes dépendent peu de la loi de comportement.

Les limites de la modélisation bidimensionnelle sont doubles : d'une part, il est délicat de définir a priori le taux de déconfinement et, d'autre part, l'évolution des contraintes dans le massif, en particulier de la contrainte déviatorique, n'est pas comparable à celle du modèle 3D.

Le calage du taux de déconfinement peut être effectué à partir d'un modèle 3D ou d'un retour d'expérience sur une grandeur en déplacement spécifique (la convergence en clé ou le tassement maximal le plus souvent). L'utilisation des formules classiques (dédiées aux tunnels profonds pour des contraintes initiales isotropes) est à éviter. Le taux de déconfinement qui permet de retrouver les tassements donnés par les calculs tridimensionnels est dépendant des caractéristiques géométriques et de creusement du tunnel, des contraintes initiales. Il dépend également de la loi de comportement et des paramètres retenus si celle-ci met en jeu une dépendance vis-àvis de la sollicitation déviatorique. Le taux de déconfinement ne peut donc pas être reconduit d'une situation à l'autre.

La différence dans les chemins de contraintes pose une autre difficulté plus difficile à surmonter. Pour une loi de comportement complexe, le fait que la modélisation 2D ne reflète pas les chemins de contraintes suivis par le sol compromet la représentativité global du calcul de tassement, dans la mesure où l'essentiel des déformations à l'origine des tassements se 
produit en avant du front et au-dessus du tunnel. En particulier, la modélisation bidimensionnelle semble mal adaptée pour prendre en compte des dispositifs de renforcement en voûte ou de présoutènement qui ont pour objet de contrôler et limiter la variation de certaines composantes des contraintes.

Ainsi, pour des lois de comportement qui dépendent de la contrainte déviatorique, l'impact des différences entre les chemins de contraintes $2 \mathrm{D}$ et $3 \mathrm{D}$ est marqué. On l'illustre dans la suite pour différentes lois de comportement, et on met ainsi en avant les limites de la modélisation bidimensionnelle.

\section{Quelques illustrations}

\subsection{Loi élasto-plastique parfaite}

L'ajout d'un critère de rupture sous sollicitation déviatorique a une conséquence sur le taux de déconfinement et les zones où la rupture est atteinte. Pour illustrer ce point, on combine la loi de comportement précédente avec un mécanisme de plasticité, le critère de rupture étant celui de Mohr-Coulomb. On considère trois couples $(c, \varphi)$ différents : $\left(40 \mathrm{kPa} ; 30^{\circ}\right)\left(35 \mathrm{kPa} ; 27^{\circ}\right)$ et $\left(25 \mathrm{kPa} ; 24^{\circ}\right)$. L'angle de dilatance est égal à $5^{\circ}$ pour l'ensemble des modèles. Avec ces paramètres, le critère de rupture est atteint dans le modèle tridimensionnel. Les travaux de Corbetta (1991) qui intègrent l'élasto-plasticité pour une formule analytique du taux de déconfinement ne sont pas adaptés à l'évaluation des tassements du tunnel superficiel. On détermine donc le taux de déconfinement en 2D par calage du tassement maximal en fin de modélisation sur celui du modèle 3D. On relève le tassement maximal en 3D et le taux de déconfinement correspondant du modèle $2 \mathrm{D}$. On relève également le tassement apporté par les déformations plastiques en 3D, et en 2D dans le Tableau 2. On isole les déformations plastiques afin de mettre en évidence l'influence de l'atteinte ou non du critère de rupture. En 2D, on a fait la différence entre le tassement maximal et le tassement maximal du modèle simplement élastique à taux de déconfinement égal.

Le taux de déconfinement dépend des caractéristiques de rupture et varie, selon le cas considéré, de 55 à $67 \%$. On relève que plus les paramètres de rupture sont faibles, plus le taux de déconfinement est élevé. Dans le cas $\left(40 \mathrm{kPa} ; 30^{\circ}\right)$, le critère de rupture est atteint en rein pour un taux de déconfinement proche du taux de déconfinement final et l'augmentation du tassement est quasi exclusivement due à l'augmentation du taux de déconfinement. Dans le cas $\left(25 \mathrm{kPa} ; 24^{\circ}\right)$, le critère de rupture est atteint pour un taux de déconfinement plus faible et la part due aux déformations plastiques est prépondérante. Dès lors, le taux de déconfinement ne modélise plus seulement la distance au front de taille mais également les déformations plastiques.

On présente sur la Figure 6 les zones dans lesquelles la norme des déformations plastiques à la pose du soutènement dépasse $0,1 \%$ et $0,5 \%$, pour les calculs $2 \mathrm{D}$ et $3 \mathrm{D}$ dans les cas $\left(40 \mathrm{kPa} ; 30^{\circ}\right)$ et $\left(25 \mathrm{kPa} ; 24^{\circ}\right)$. Le modèle bidimensionnel ne prévoit pas de zone plastifiée au-dessus du tunnel quel que soit le cas : on pourrait être tenté d'en conclure que la mise en place d'un renforcement par voûte parapluie ou boulonnage radial en voûte n'aurait pas d'influence sur les tassements. La modélisation tridimensionnelle ne tend pas à la même conclusion puisque la plastification est présente tout autour
Tableau 2. Tassement maximal et taux de déconfinement en fonction des paramètres de rupture.

Table 2. Maximum settlement and stress relaxation factor versus failure criteria.

\begin{tabular}{lllll}
\hline & $\begin{array}{l}\text { Élastique } \\
\text { linéaire }\end{array}$ & $\begin{array}{l}c=40 \mathrm{kPa} \\
\varphi=30^{\circ}\end{array}$ & $\begin{array}{l}c=35 \mathrm{kPa} \\
\varphi=27^{\circ}\end{array}$ & $\begin{array}{l}c=25 \mathrm{kPa} \\
\varphi=24^{\circ}\end{array}$ \\
\hline $\begin{array}{l}S_{\max }(\mathrm{mm}) \\
\lambda(\%)\end{array}$ & 5,1 & 9,2 & 11,1 & 17,6 \\
$\begin{array}{l}\text { Part plastique } \\
\text { 3D (mm) }\end{array}$ & - & 55 & 61 & 67 \\
$\begin{array}{l}\text { Part plastique } \\
\text { 2D (mm) }\end{array}$ & - & 4,1 & 6,0 & 12,5 \\
\hline
\end{tabular}

de la section à la hauteur du front de taille. Ainsi, une analyse de stabilité de l'excavation sur la base de l'étendue de la zone plastique conduirait à des conclusions différentes selon la modélisation considérée bien que le tassement maximal calculé soit identique.

La difficulté d'anticiper le taux de déconfinement et l'écart dans l'étendue des zones plastiques fragilise l'utilisation de la méthode bidimensionnelle dans le cas où le critère de rupture est atteint.

\subsection{Loi élastique non linéaire}

On présente ici de nouvelles simulations, dans lesquelles on attribue au sol une loi élastique non linéaire dont la principale caractéristique est de faire dépendre le module d'élasticité tangent avec la contrainte déviatorique normalisée par sa valeur initiale, suivant (Éq. (4)). On trouvera plus d'informations sur cette formulation dans la référence (Gilleron et al., 2016a) :

$$
G_{t}=\operatorname{Min}\left(\operatorname{Max}\left(G_{\min } ; G_{0} \xi^{1-\frac{q}{q_{i}}}\right) ; G_{0}\right),
$$

où $G_{\min }$ désigne le module de cisaillement minimal, $G_{0}$ le module de cisaillement initial, $\xi$ est un coefficient de forme qui décrit la décroissance de la raideur lorsque le déviateur augmente et $q_{i}$ est la contrainte déviatorique initiale dans le massif. Le coefficient $\xi$ est un paramètre du modèle qui contrôle la non-linéarité du module (si $\xi$ vaut 1 , le modèle est linéaire). Le coefficient de Poisson est défini de telle manière que le module de compression isotrope est constant suivant l'idée proposée par Fahey et Carter (1993). Le coefficient de Poisson croît à mesure que le module de cisaillement décroît. On présente sur la Figure 7 les variations du module de cisaillement tangent et du coefficient de Poisson tangent en fonction de la contrainte déviatorique.

On retient $G_{0}=45 \mathrm{MPa}, G_{\text {min }}=4,5 \mathrm{MPa}$ et le coefficient de Poisson initial égal à 0,1 . Pour ces paramètres, le module de Young à l'état initial est égal à $100 \mathrm{MPa}$ dans tout le massif.

À l'aide de cette formulation, on détermine le taux de déconfinement par calage du tassement maximal pour différentes valeurs de $\xi$. On relève le tassement maximal et le taux de déconfinement dans le Tableau 3.

Tous les autres paramètres étant fixés, plus la sensibilité à la contrainte déviatorique est prononcée ( $\xi$ est élevé), plus le 

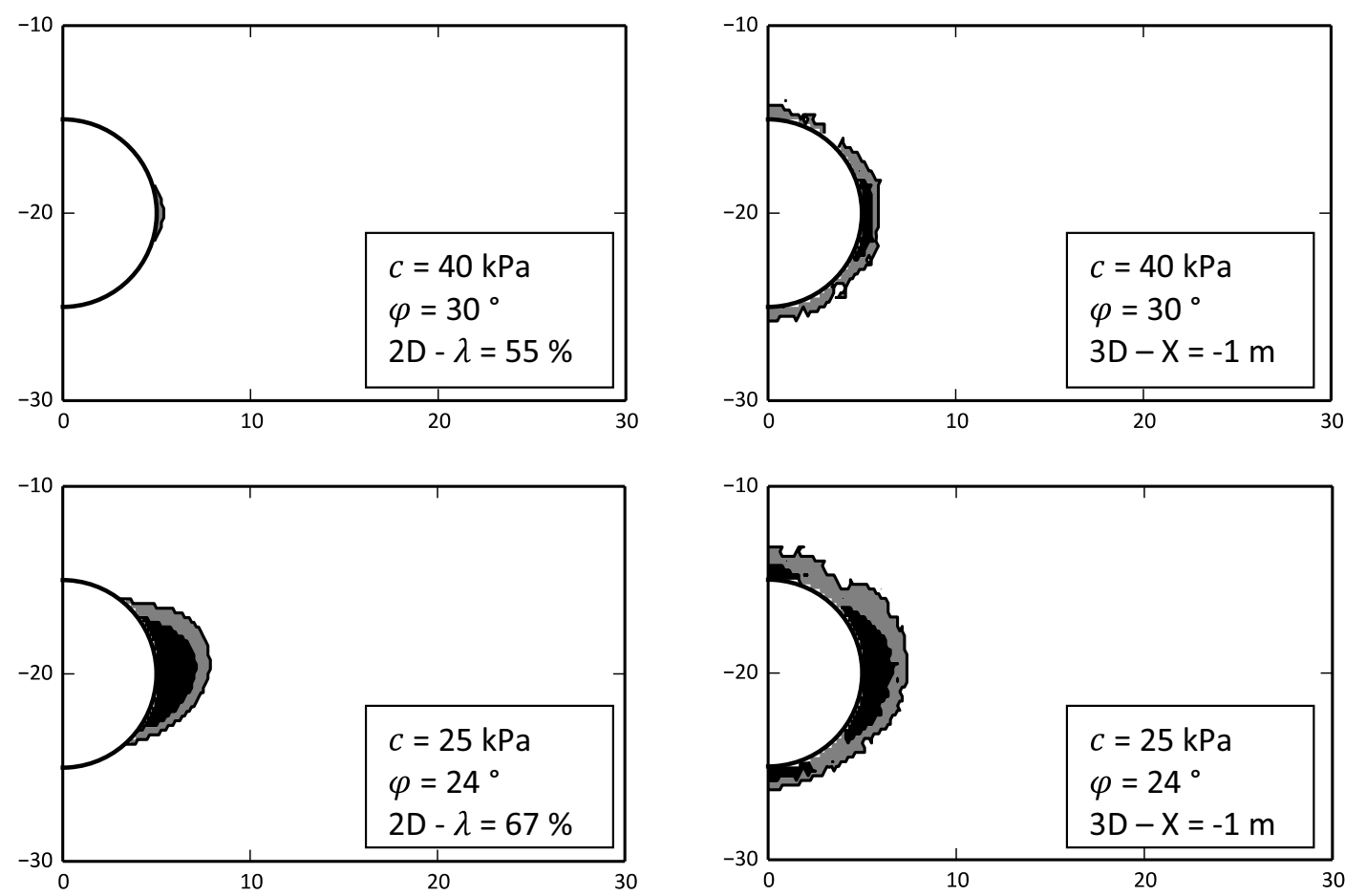

Fig. 6. Zones où la déformation plastique est supérieure à $0,5 \%$ (noir) et $0,1 \%$ (gris) pour différents paramètres de rupture en $2 \mathrm{D}$ et en $3 \mathrm{D}$. Fig. 6. Areas where plastic strains exceed $0.5 \%$ (black) and $0.1 \%$ (grey) for two failure criteria in $2 D$ et $3 D$.
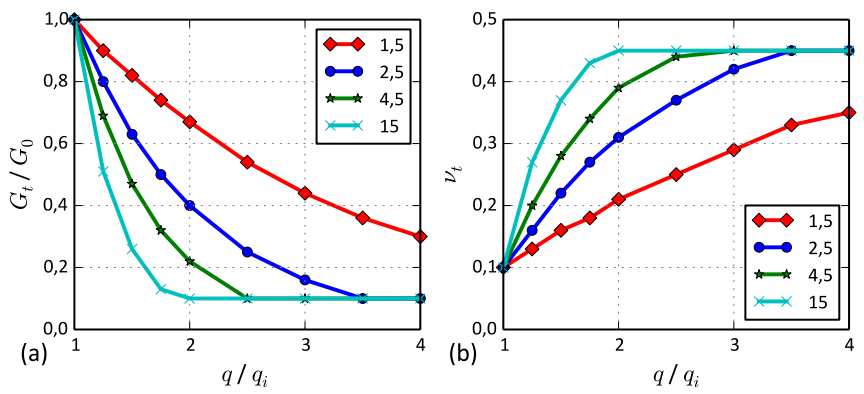

Fig. 7. Variation des paramètres élastiques avec la contrainte déviatorique pour différentes valeurs de $\xi$ (a) Module de cisaillement tangent (b) Coefficient de Poisson tangent pour $v_{0}=0,1$.

Fig. 7. Elastic parameters variation versus deviatoric stress for different values of $\xi$ (a) Tangent shear modulus (b) Tangent Poisson's ratio with $v_{0}=0.1$.

Tableau 3. Tassement maximal et taux de déconfinement en fonction du paramètre $\xi$.

Table 3. Maximum settlement and stress relaxation factor versus $\xi$ parameter.

\begin{tabular}{lllll}
\hline$\xi(-)$ & 1,5 & 2,5 & 4,5 & 15 \\
$S_{\max }(\mathrm{mm})$ & 6,8 & 10,0 & 15,1 & 25,1 \\
$\lambda(\%)$ & 40 & 50 & 57 & 61 \\
\hline
\end{tabular}

taux de déconfinement est élevé. D'autre part, le tassement maximum ne varie pas linéairement avec le taux de déconfinement, la non-linéarité introduite dans le modèle est mise en jeu dès que la contrainte déviatorique commence à croître. Le taux de déconfinement dépend du profil de l'élasticité non linéaire utilisé.
On présente sur la Figure 8 les isovaleurs du module d'élasticité tangent avant la pose du soutènement pour $\xi$ égal à 4,5. Là encore, la situation est très différente entre les modélisations $2 \mathrm{D}$ et $3 \mathrm{D}$ : on observe en particulier que la modélisation $2 \mathrm{D}$ conduit à une zone plus raide (où le module est sensiblement égal à sa valeur initiale) au-dessus de la clé du tunnel qu'on ne trouve pas dans le calcul 3D.

\subsection{Loi Hardening Soil Model}

Avec une loi de comportement comme le Hardening Soil Model, décrite par Schanz et al. (1999), on obtient, dans les calculs $2 \mathrm{D}$, une zone plastique qui se développe à partir du rein, tandis que la zone située au-dessus de la clé de voûte reste dans le domaine élastique (Fig. 9). Le module tangent dans cette zone en 2D est alors égal au module de rechargement/ déchargement, ce qui conduit à surestimer sa raideur par rapport au calcul 3D (Gilleron et al., 2016b).

Comme pour les autres modèles de comportement, le calage du taux de déconfinement pour obtenir le tassement passe par l'établissement d'un modèle 3D, et la reprise d'un calage issu d'un calcul différent ne donne pas de garantie. D'autre part, la multiplicité des mécanismes et des paramètres du modèle HSM rend difficile de prévoir l'évolution du taux de déconfinement en fonction des paramètres de la loi de comportement, comme on a pu le faire pour les modèles précédents.

\section{Conclusions}

L'évolution du déviateur des contraintes dans le terrain autour d'un tunnel est différente dans un calcul bidimensionnel et dans un calcul tridimensionnel. Cela explique pourquoi la valeur du taux de déconfinement qu'il faut adopter pour 

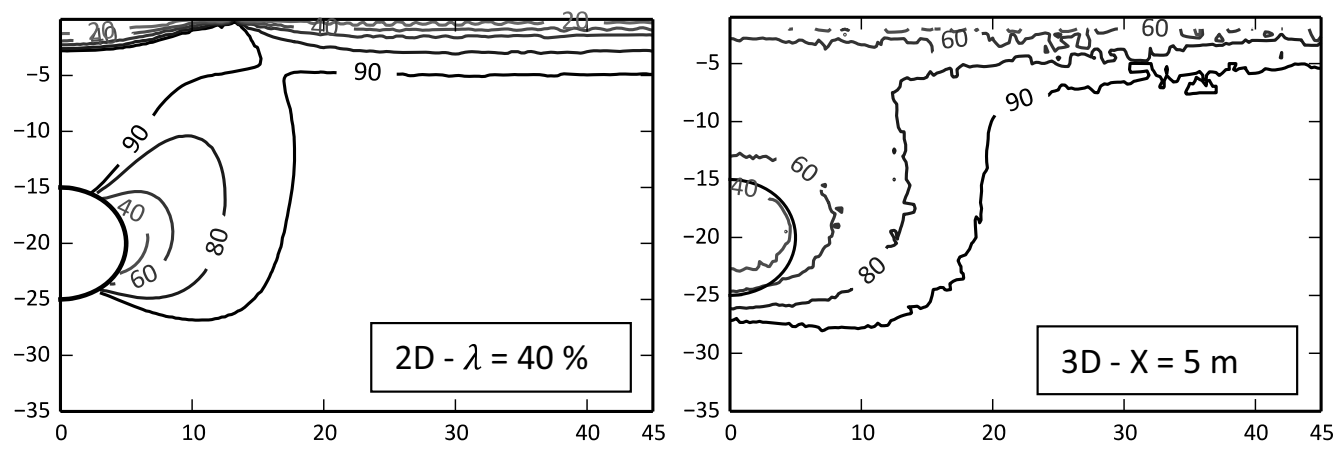

Fig. 8. Isovaleurs de module de Young tangent - cas $\xi=4,5$ en $2 \mathrm{D}$ et en $3 \mathrm{D}$.

Fig. 8. Contour lines of tangent Young's Modulus in $2 D$ and $3 D$.
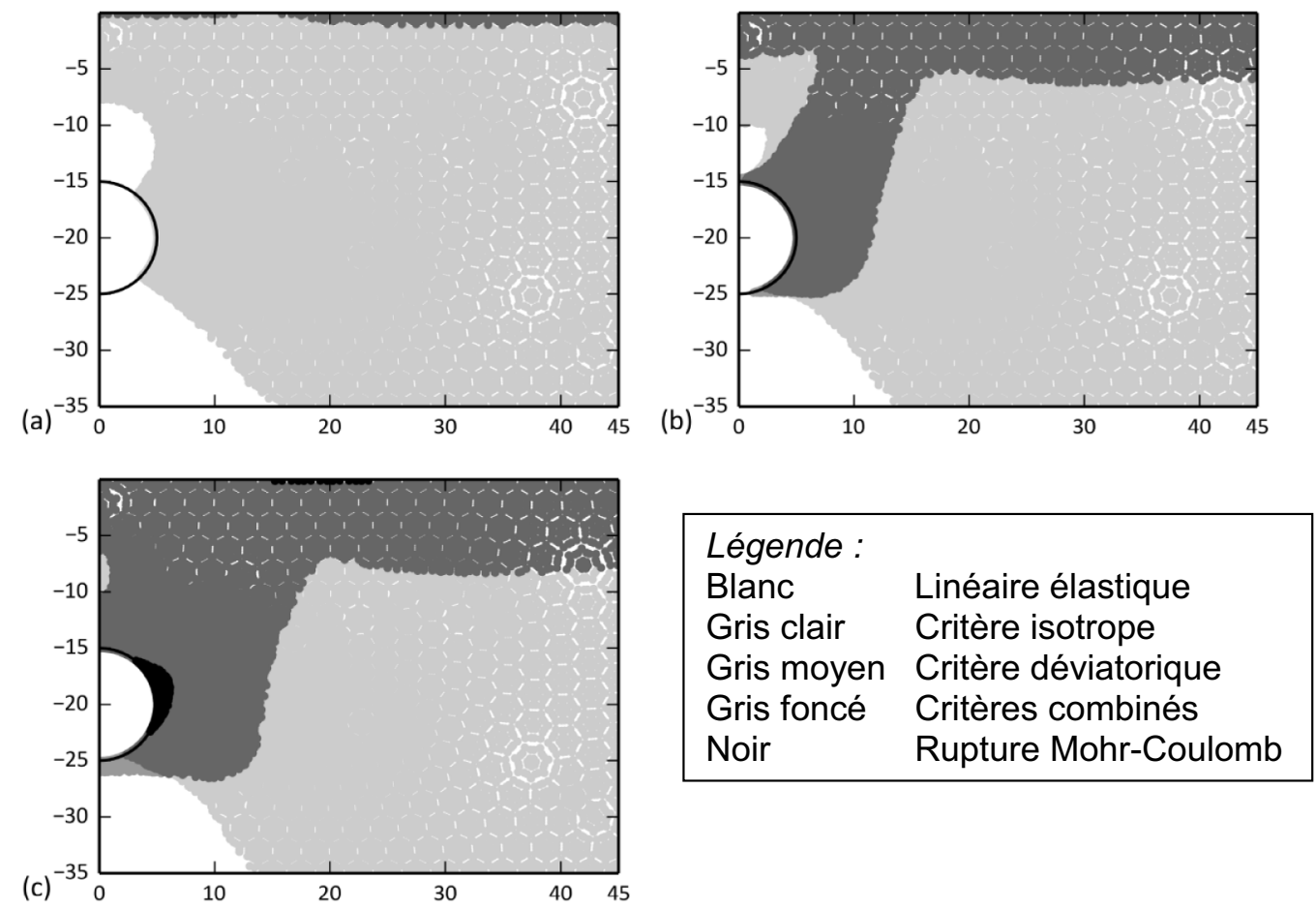

Fig. 9. Zones plastiques en fonction du taux de déconfinement (a) $10 \%$ (b) $50 \%$ (c) $65 \%$, extrait (Gilleron et al., 2016b).

Fig. 9. Plastic areas with stress relaxation factor (a) $10 \%$ (b) $50 \%$ (c) $65 \%$, from (Gilleron et al., 2016b).

retrouver avec un calcul en déformations planes les mêmes tassements qu'avec un calcul tridimensionnel dépend étroitement de la loi de comportement du sol. On peut aussi souligner que, même si le taux de déconfinement est choisi de telle sorte que les tassements maximaux sont égaux, la différence des états de contraintes réduit de beaucoup la confiance qu'on peut avoir dans une modélisation bidimensionnelle qui chercherait à prendre en compte des procédés de présoutènement en voûte du tunnel. De manière générale, cette étude remet en question la pertinence de l'approche bidimensionnelle par relaxation des contraintes pour le calcul des tassements en surface.

Remerciements. Les auteurs remercient le Fond Unique Interministériel, le CG78 et BPIFrance pour leur support au projet Newtun.

\section{Références}

Bernaud D, Rousset G. 1992. La nouvelle «méthode implicite » pour l'étude du dimensionnement des tunnels. Rev Fr Geotech 60: 5-26.

Fahey M, Carter JP. 1993. A finite element study of the pressuremeter in sand using a nonlinear elastic plastic model. Can Geotech J 30: 348-362.

Gilleron N, Bourgeois E, Saïtta A. 2016a. Influence of deviatoric stress dependent stiffness on settlement trough width in 2D and 3D finite element modelling of tunnelling. In: Proceedings of the 17th Nordic Geotechnical Meeting, Reykjavik.

Gilleron N, Bourgeois E, Saïtta A. 2016b. Utilisation du hardening soil model dans l'étude du creusement de tunnels superficiels. In : Journée nationales de géotechnique et de géologie de l'ingénieur, Nancy. 
Humbert P, Dubouchet, A, Fezans G, Remaud D. 2005. CESARLCPC, un progiciel de calcul dédié au génie civil. Bulletin des Laboratoires des Ponts et Chaussées 256: 7-37.

Janin J, Dias D, Emeriault F, Kastner R, Le Bissonnais H, Guilloux A. 2015. Numerical back-analysis of the southern Toulon tunnel measurements: a comparison of 3D and 2D approaches. Eng Geol 195: 42-52.

Möller S, Vermeer P, Bonnier P. 2003. A fast 3D tunnel analysis. In: 2nd MIT Conference on computational fluid and solid mechanics, pp. 486-489.
Panet M. 1995. Le calcul des tunnels par la méthode convergenceconfinement. Paris : Presses de l'école nationale des Ponts et Chaussées.

Schanz T, Vermeer PA, Bonnier BG. 1999. The hardening soil model: formulation and verification. In: Beyond 2000 in Computational Geotechnics. Balkema: Rotterdam, pp. 281-290.

Wedekin V, Kastner R, Guilloux A, Bezuijen A, Standing J, Negro Jr A. 2012. Urban tunnels in soft ground: review of current design practice. In: Proceedings Geotechnical aspects of underground construction in soft ground, Taylor \& Francis Group, London, pp. 1047-1064.

Citation de l'article : Nicolas Gilleron, Emmanuel Bourgeois, Adrien Saïtta. Limites de la modélisation bidimensionnelle des tunnels urbains pour la prévision des tassements. Rev. Fr. Geotech. 2017, 150, 2. 